\title{
Smart Real-Time Healthcare Monitoring and Tracking System using GSM/GPS Technologies
}

\author{
Saed Tarapiah \\ Telecommunication Engineering Dept. \\ An-Najah National University \\ Nablus, Palestine
}

\author{
Kahtan Aziz \\ College of Engineering Computing. \\ Al Ghurair University \\ Dubai, United Arab Emirates
Salah Haj Ismail
Faculty of architecture
Aleppo University
Aleppo, Syria

\author{
Shadi Atalla \\ College of Information Technology (CIT) \\ University of Dubai \\ Dubai, United Arab Emirates
}

\begin{abstract}
Recently, remote monitoring systems have evolved to respond for particular needs in healthcare sector, which is an essential pillar in the modern concept of smart city, we propose a smart system to monitor patient current health conditions, as a smart healthcare system based on the widely spread available technologies; namely, GSM and GPS. Statistics shows that hypertensive heart disease and blood pressure are risk factors for high death rate to decrease it a preventive measures should be applied providing a real-time health monitoring system, to save patients life at acceptable time. The objectives of this paper is to provide an effective system model, that will track, trace, and monitor patient vital readings in order to provide efficient medical services in time. By using sensors, the data will be captured and compared with a predefined threshold. The study focuses on heartbeat rate, and body temperature, thus in case of emergency an SMS will be sent to the Doctors mobile containing measured values and position. Moreover, the paper demonstrates the possibility of building a complete end-to-end smart healthcare monitoring system by using wide range of available sensors for more vital human health parameters to connect patient with doctors in cases of emergency.
\end{abstract}

\section{Keywords}

Smart Healthcare, Sensors, Smart City, Embedded Systems, Monitoring.

\section{INTRODUCTION}

According to international worldwide data of the global effects of hypertension heart diseases [10], the analysis study demonstrates that blood pressure or hypertension affects more than 1 billion people worldwide. Rather than the hypertension heart diseases, the high blood can be a factor and attribute to cause many other disorders, such as stroke aneurysms, ischemic heart, and kidney disease. The risk of heart failure, due to Hypertension is almost increased by factor two or three-fold [13], and may accounts for about $25 \%$ of all heart failure cases [9]. Moreover, the hypertension or high blood pressure in $90 \%$ of cases preceded or advanced the heart failure telescopically for elderly. Hypertension was ranked 13th in the leading global causes of death for all ages [12]. A world map shows the distribution of diseases caused by high blood pressure in Figure 1 Statistics for the numbers of heart diseases were not available due to the bad condition of many countries of the middle east region, so this project may encourage relevant institutions to collect such statistics.

In an era of laziness and lack of physical exercises, fat, sugar, meats, smoking, obesity, and the lack of eating fruit and vegetables. Under the shade of difficult economic situation, physical fatigue, stress and to having dignified life the blood pressure disease has been the famous one in the last century due to poor eating habits. These neurological conditions are chief of this kind of illness, in many countries. Not only medical intervention is required to detract the effects but Smart and Innovative solutions should be adopted to increase the responsive actions quality in both time and level; and since Sarwant Singh [14] has identified eight key aspects that define a smart city as shown in figure 2 which depicts the smart city concepts: smart governance; smart building; smart infrastructure; smart energy, smart technology; smart citizen; smart mobility; and smart healthcare. In this paper we propose a system architecture for smart healthcare based on GSM and GPS technologies.

Based on visiting hospitals, it was approved of the effectiveness of this project and its ability to facilitate communication between the patient and his doctor. Utilizing the available services of GSM and GPS technologies to build a smart health monitoring system can improve and enhance the real time monitoring, where: GSM services are used for global communications any time and anywhere, GPS technology is applied for outdoor positioning, many works has been carried to use GPS and SMS for localization and reporting as stated in [17] [21] [4].

Figure 3 describes the flow chart of the suggested system, starting with reading the heart rate and body temperature by using specific sensors: pulse sensor and temperature sensor; the captured data will be compared via microcontroller i.e. Arduino with a given threshold. The Arduino also keep checking the position twenty times using the GPS module. The readings will be compared with the maxi- 


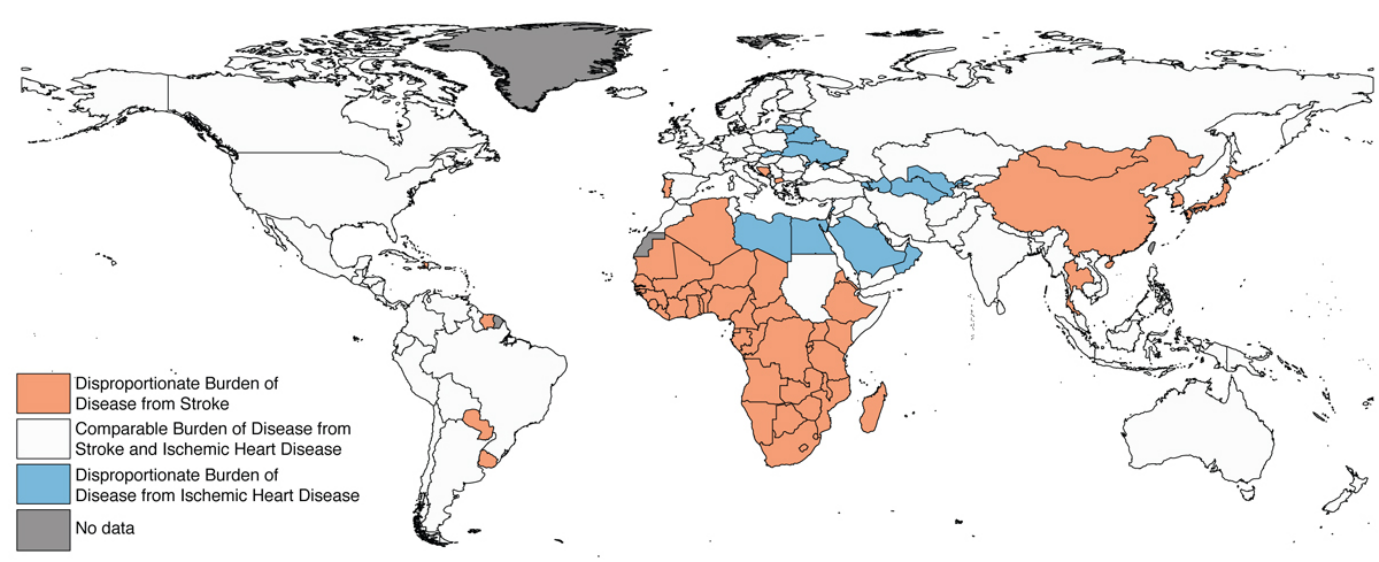

Fig. 1. Distribution of Diseases Caused by High Blood Pressure [11

\section{SMART CITY CONCEPTS}

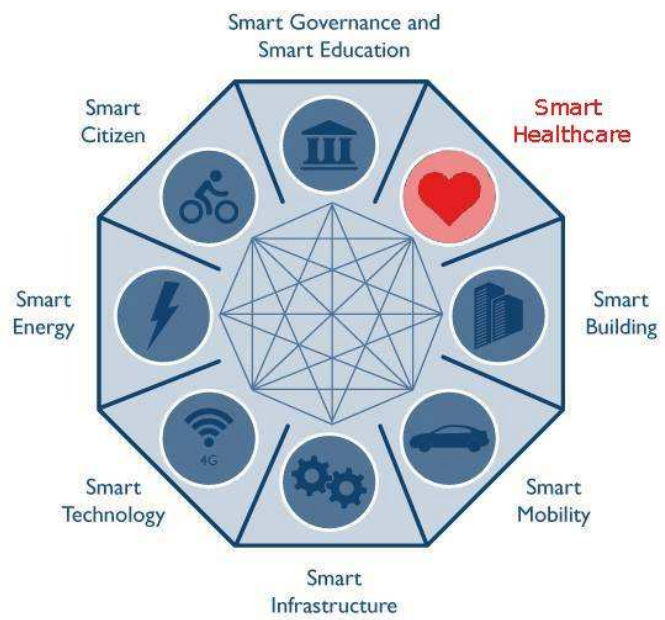

Fig. 2. Smart City Concepts

mum and minimum stored values in the microcontroller, in the case that the measured values where out of the allowed threshold range a SMS will be sent immediately to the relevant person contains: the patient name, heart rate, body temperature, the patient's location and the corresponding UTC time-stamp. Electrocardiogram (ECG) could be drawn by sending the reading to a specialized processor. Our earlier related work is discussed in [3] [19] [20].

The paper is organized as the following: Section 1 introduces the importance of the Smart Health system. Section 2 states the different emerging engineering issues. Section 3 provides a brief summary of similar research projects and papers. Section 4 presents insights structure of the proposed system and explains the main building blocks and the interconnection relationships among the system blocks. Preliminary results and their discussion are collected using the implemented parts of the proposed system are shown in Section 5 Finally, Section 6 conclude the paper and presents the future plans to enhance the proposed system.

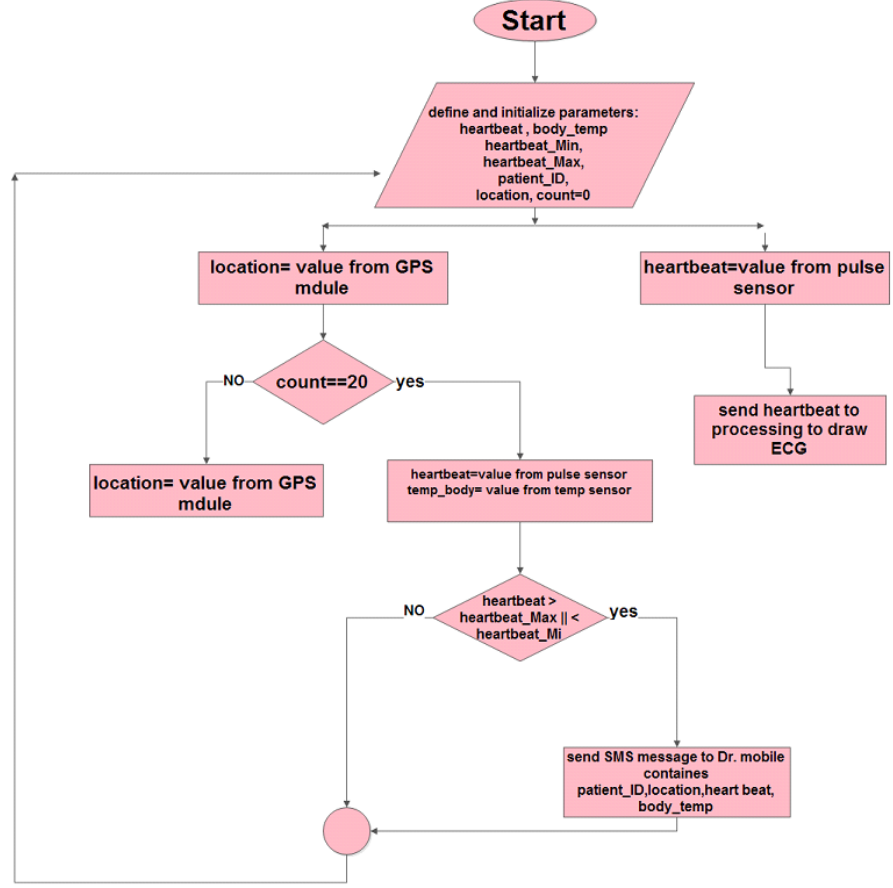

Fig. 3. System Flow-Chart

\section{ETHICAL, SOCIAL AND ENGINEERING ISSUES}

In the era of globalization, the proposed system model, could be implemented in different countries with different cultures and big diversity of legal and political enforcement. and although the paper is focusing on the technical and engineering issues from ICT perspectives, we can't overlook the issues created by its global possible implementations such as:

-Social and political issues: The system would create notable social impact by questioning the new relation between the patient and the doctor, and its effect on the medical work life style since the doctor will be always aware of the patient's current health condition by monitoring his vital readings through the proposed 
model any time, and any where. Moreover, the legal responsibilities should be defined in cases of system down, or misbehavior, by ratification new regulations and laws to control and manage such cases.

-Ethical issues: The privacy dilemma for both patient's location and vital readings should be discussed with those who will be objects for the implementation and using the system, with different culture which may not accept such system functions in term of surveillance. Real time health monitoring systems, however, are only the foundation tool. Doctors, patients and any other potential system beneficiary must be educated about real time health monitoring in order to ensure that all of them perceive the privacy issues posed by such system. Moreover, Doctors and patients need to be educated to understand how the proposed system and related technologies works, to be able to spot the system capabilities as well as limitations.

- Security issues: The digital immunity of the system relies on basic security mechanism such as authorization and authentication provided, moreover the GSM technology is a secure and licensed technology by its standards. Deep inspection and security procedures must be carried out to ensure the system equipped with advanced and high security standards.

-Sustainability issues: Although, the system is composed of low maintenance set of electronics, it can't last functioning for ever due to its physical nature, so the system needs to consider periodic tests and inspections for realize system high sustainability, moreover, ICT system administrations is required to guarantee the system reliability. Notwithstanding, the system components should take into consideration low energy consumption.

-Development issues: Healthcare sector needs both new applications using already existing components and development of new electronic components or software which enable a wider range of application possibilities in the healthcare monitoring systems.

-Manufacturing issues: Enhancement in healthcare systems needs the suggested and proposed new systems to be manufactured and implemented in real life, even by impeding these technologies in smart phones and wearable devices.

\section{RELATED WORK}

Mikhail St-Denis,designed Life line project that can monitor heart rate, blood sugar levels, human's body temperature, and by using a wireless communication technologies to synchronize and display these information into a smart mobile phone or a standard computer. such device gather data from user and display some related graphs in order to encourage users to remain aware of their health conditions by providing a week to week feedback [15].

Eli Hariton, designed Gluco (M) wristband which monitors the blood glucose levels [5]. LUMO BodyTech(2011), created a platform for tracking human biomechanics, starting with a unique sensor-based solution for posture and back pain. This solution is comprised of a discreet biomechanics-monitoring sensor, an engaging mobile app, and intelligent algorithms for a personalized user experience. Patent-pending solution harnesses the power of human movement data to provide real-time actionable feedback and to enable healthy behaviors [2]. Dr. Sailesh Chutani(2009), founded a Mobisante for ultrasound imaging that will be displayed. Health care workers in remote locations can check pregnant women, monitor a baby's health, examine patients for heart and lung problems, and triage other problems. Their phone can then transmit the images to a hospital for consultation [24]. In this paper, a tracking system will be designed and implemented for monitoring heart rate and body temperature.

\section{ARCHITECTURE AND IMPLEMENTATIONS}

This section provides insights structure of the proposed system and explains the main building blocks and the interconnection relationships among the system blocks. Mainly, the proposed system aims to cover an end-to-end smart health application that can be build up from two functional building blocks. However the main function of the first building block is to gather all sensory data that are related to the monitored persons, whereas the second block functions are to store, process and present the resulted information of this stage to the doctors and nursery staff that are fallowing the case of the monitored person.

As depicted in Figure 4 , which illustrates the overall model, when the patient's heartbeat rate changes badly, the Arduino which recorded Pulse and LilyPad Temperature Sensors readings, orders GSM shield to send an SMS message containing these readings, patient ID and the location of the patient which has been taken via GPS shield, to his doctor's mobile phone, who -by his turn- send an ambulance to the patient's location.

\subsection{Smart Embedded Board (SEB)}

This subsection provides the hardware components details used to compose a smart board attached to the human body. Periodically, the Smart board senses the human health conditions using several dedicated sensor devices and then the broad conveys the raw sensed data to the back-end server application using GSM SMS.

4.1.1 Microcontroller. It is the core part of the SEB design; the microcontroller acts as the brain of the smart board that is holding the main board flow chart logic. However, there are many microcontrollers available in market and can perform well the main board logic such as PIC, Beagle-Bone, and Arduino. For the sake of demonstration proposes the choice falls on Arduino Uno according to its specifications and simplicity of use. Arduino Uno as depicted in Figure 5 this board is based on ATmega32 microcontroller, which has a set of 14 input/output digital pins, where 6 out of 14 can be used as a PWM output pins, also, the microcontroller board has 6 analog inputs, a ceramic resonant of $16 \mathrm{MHz}$, an USB interface, a DC power jack, a reset button, and ICSP header. The USB interface, simplifies the connection of the microcontroller with the computer, also the USB can be a power supplier for the microcontroller board [25].

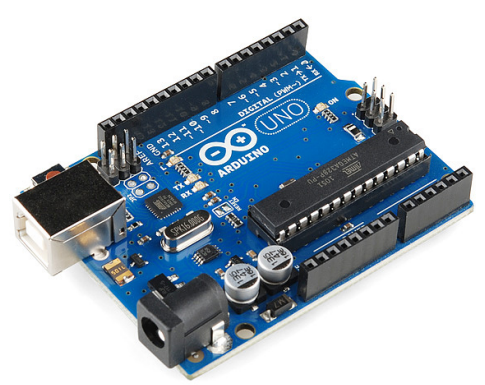

Fig. 5. Arduino Microcontroller 


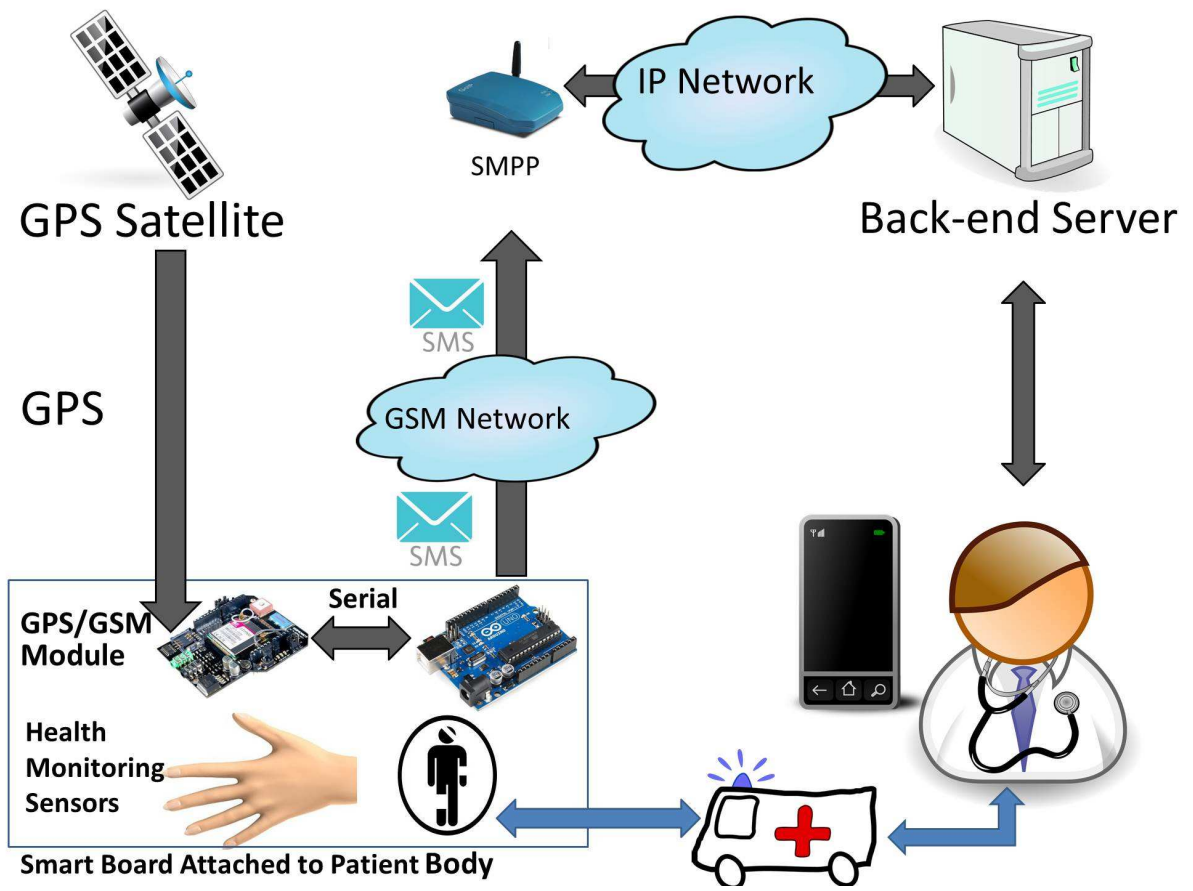

Fig. 4. Proposed System Model

4.1.2 GPS/GPRS/GSM MODULE V3.0. This is a GPS / GPRS / GSM shield from DFRobot as depicted in Figure 6 is a Quadband GSM / GPRS engine that works on frequencies EGSM $900 \mathrm{MHz} / \mathrm{DCS} 1800 \mathrm{MHz}$ and GSM850 MHz / PCS 1900MHz. It is also Supports GPS technology for satellite navigation.[14] Sending messages via GSM network controlled via AT commands (GSM07.07, 7:05 and SIMCOM enhanced AT Commands). The design of the shield allows driving the GSM and GPS function directly with any computer and Arduino board. GPS/GPRS/GSM shield includes a high-gain SMD antenna for GPS and GSM. The consumption expenditure of SIM548C is an embedded chip from SIMCom [7].

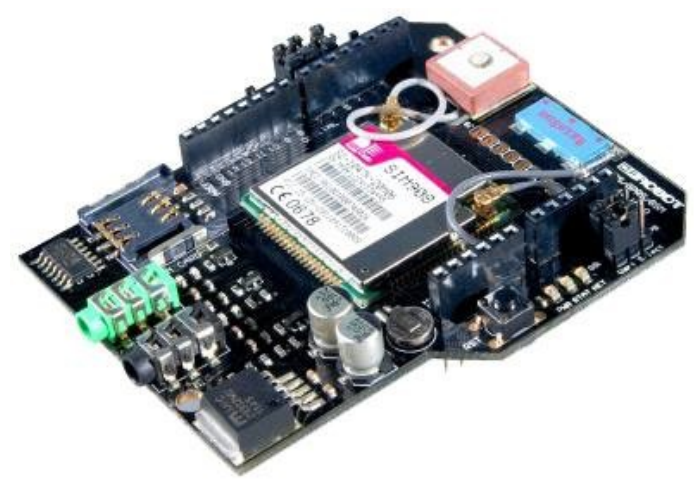

Fig. 6. Dfrobot GPS/GPRS/GSM MODULE V3.0
4.1.3 Heart Beat pulse Sensor. Figure 7 shows the heart beat pulse rate sensor, whereas the pulse measurement is not an easy task;pulse sensor measures the heart rate optically, amplifies the signal and eliminate the noise by connecting the sensor directly to Arduino or any other controller with working voltages from 3 to $5 \mathrm{~V}$. Simply plug the sensor on the ear or finger sensor and consider that the maximum wire length of about $60 \mathrm{~cm}[1]$.

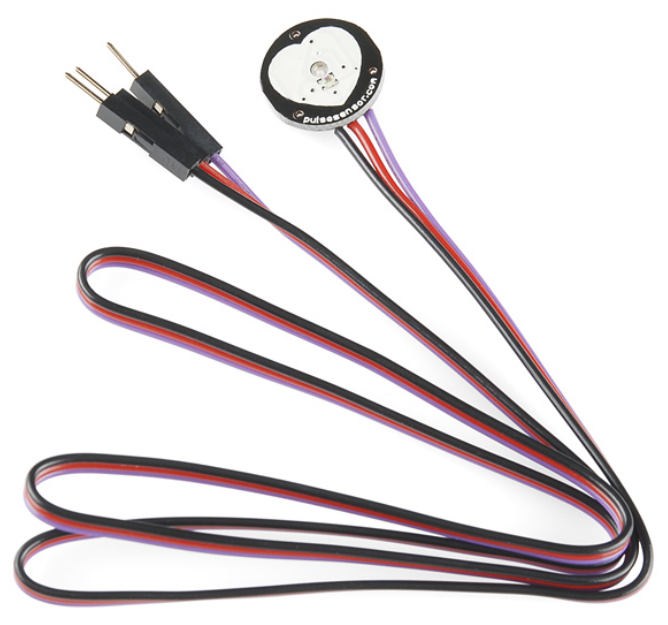

Fig. 7. Heart Beat Pulse Sensor 
4.1.4 Human Body Temperature Sensor. Detecting temperature changes has become easier using MCP9700 which is a small thermostat type temperature sensor. The output will be $0.5 \mathrm{~V}$ at 0 degrees $\mathrm{C}, 0.75 \mathrm{~V}$ at $25 \mathrm{C}$, and $10 \mathrm{mV}$ per degree C. Doing an analog to digital conversion on the signal line will allow to establish the local ambient temperature. Detect physical touch based on body heat and ambient conditions with this small sensor. Also LilyPad as depicted in Figure 8 is a wearable e-textile technology developed by Leah Buechley and cooperatively designed by Leah and SparkFun where each LilyPad was creatively designed to have large connecting pads to allow them to be sewn into clothing. Moreover, various input, power, output and sensor boards are available. They are even washable [6].

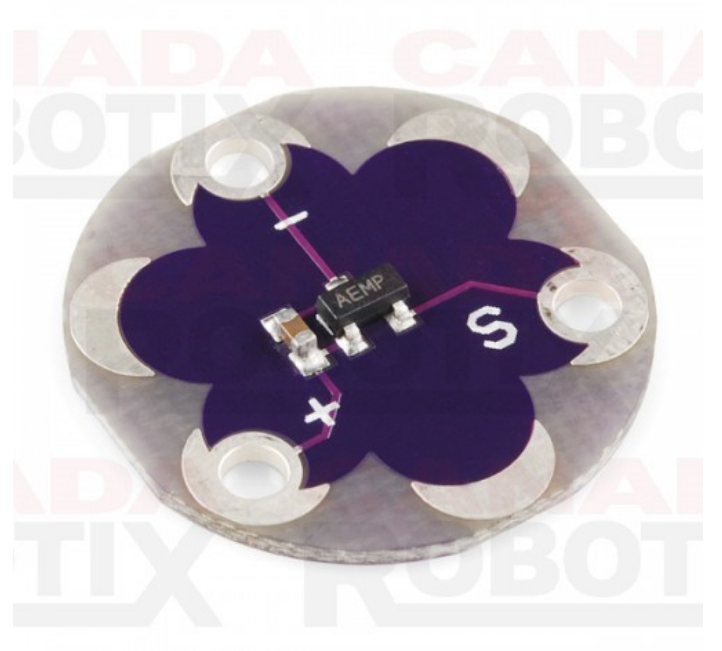

Fig. 8. LilyPad Temperature Sensor

\subsection{Online-Web Based Monitoring Application}

Doctors and Nurses are provided with a simple web-based application to track and monitoring the patient's health conditions. The implemented web application is accessible through a standard web browser, smart phone and tablets devices. The REST (RESTful) software architectural style has been adapted to insure the resulted web application is scalable and flexible. Moreover, communication among the web application modules uses JSON data representation. Furthermore, the implemented system leveraging on the wellknown three-tier architecture [8]:

(1) The front-end represents the web-page which is accessible by the Doctors and Nurses. This part uses several web technologies such as HTML5 (Hypertext Transfer Markup Language version 5), CSS (Cascading Style Sheet), the open source JQuery software library and Javascript client side programming language. However, a bidirectional data communication channel is maintained between this tire and the middle tire through the Asynchronous JavaScript AJAX technology. The final web-page is responsive and is running on smart phones, tablet devices and standard PCs.

(2) The middle tire which hosts the main server logic has been developed using PHP programming language and this logic has been deployed on an Apache web server. This tire uses

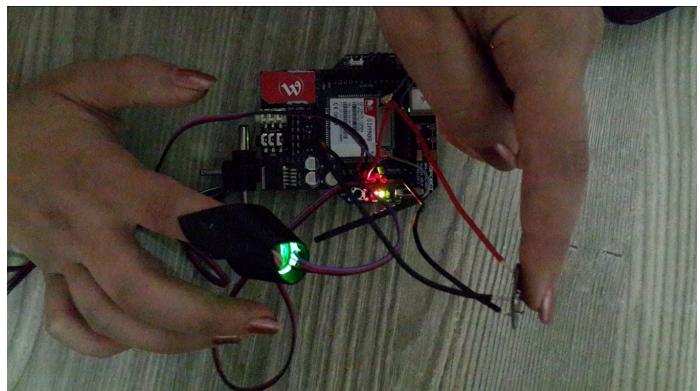

Fig. 9. System Hardware Model

RESTful style to expose its internal functionality towards the client side web-page as well as this software tire leverage on the MySQL native driver for PHP in order to store and retrieve data.

(3) The back-end tire which hosts the MySQL database server and this database is used to store all the patient data, system users(doctors, patients and nurses, patients medical profiles and their corresponding alerts. This design of this module is based on a relational database structure. However, health data records and patients' positioning information are time stamped using the standard UTC reference time.

Ina RESTful software architectural style every thing is a resource and for each resource there is a URI (Universal Resource Identifier) that represents the corresponding resource unique address. Moreover, there are four verbs that are usable to transfer and manipulate any resource representation. Finally, the word CRUD refers to these four verbs and the $\mathrm{C}$ letter is coming from create, $\mathrm{R}$ from read, $\mathrm{U}$ from update and $\mathrm{D}$ from delete. many works has been carried as in [23] [22] [18], where smart boards based on controller and related sensors are defined for specific applications.

\section{RESULTS AND DISCUSSION}

A well functioning system prototype was build composed of the following hardware components: LilyPad Temperature Sensor, Pulse Sensor, GPS / GPRS / GSM MODULE V3.0 and the Arduino integrated together to perform a healthy system as shown in Figure 9

Figure 10 shows the interfacing circuit between the temperature sensor and Arduino microcontroler. For achieving an accurate and precise patient's temperature readings the system developers work with Steinhart equation which models the resistance of semiconductor (temperature sensor) at different temperatures. For simplicity and prototype purposes the computed temperature values are based on beta factor method instead Steinhart equation [16].

Table 1 illustrates results of in site experiments on both the prototype and traditional sensors -Heartbeat and Temperature- used by doctors in real life to diagnostic patients' health conditions. For both sensors -Heartbeat and Temperature- used in the prototype the observed readings are similar to the corresponding sensor reading used by in site doctors with a minor error percentage that is tolerated for both cases, Heartbeat and Temperature readings.

In order to analyze, test and validate the system demonstrator several experiments has been performed and the results presented in Figure 11 shows the ECG -which had been drawn using simulation software- for a healthy normal persons. It is clear that the normal heart rate is in the range of 60-90. 
Table 1. Error Percentage: System Readings vs Standard Device Readings.

\begin{tabular}{|l|l|c|c|}
\hline Heartbeat / Temperature & System Reading & Standard Device Reading & Error Percentage \\
\hline HeartBeat & 86 & 84 & 2.3 \\
\hline HeartBeat & 78 & 77 & 1.2 \\
\hline Temperature & 36.91 & 37.2 & 0.78 \\
\hline Temperature & 36.46 & 36.1 & 0.83 \\
\hline
\end{tabular}

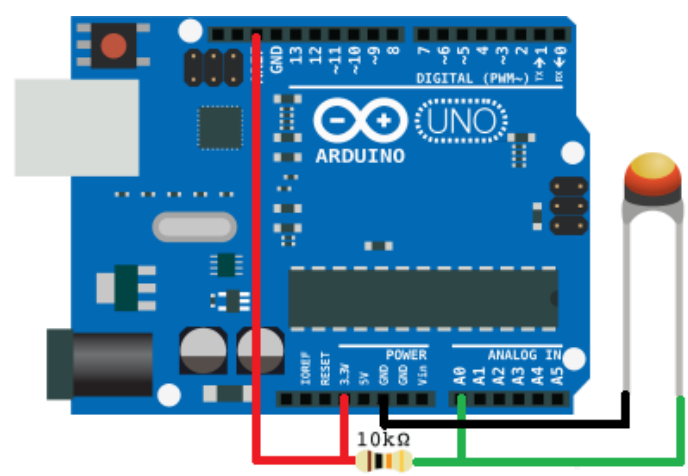

Fig. 10. Temperature Sensor Interfacing Circuit based on Beta Factor Circuit Method

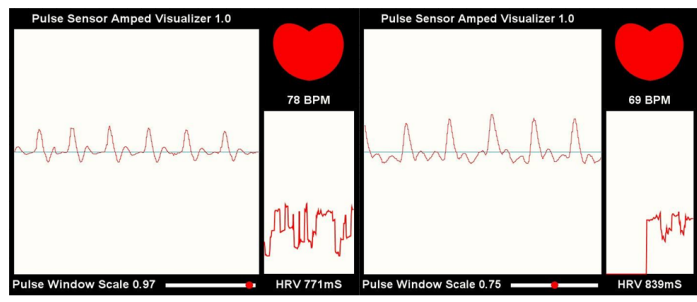

Fig. 11. Normal ECG

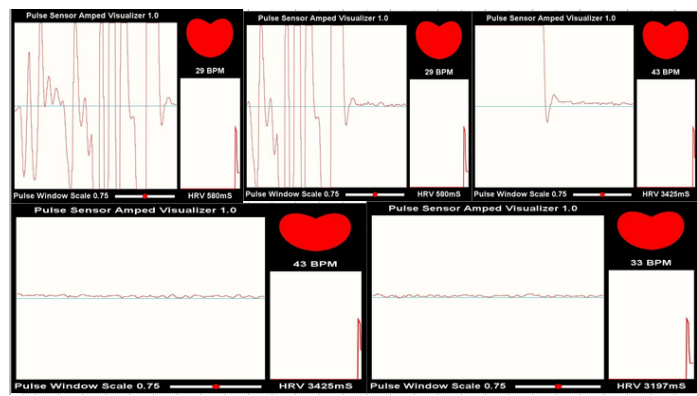

Fig. 12. Unconnected Pulse Sensor ECG

While Figure 12 shows the ECG when the sensor was unconnected to the human body.

In the other hand Figure 13 demonstrate the position (geographical position longitude and latitude) which was collected using GPS/GPRS/GSM module.

While the LilyPad Temperature Sensor readings are illustrated in Figure 14 in terms of corresponding voltage level and temperature degrees both in Celsius degree and Fahrenheit degree. Finally, all these results are displayed on the serial monitor.

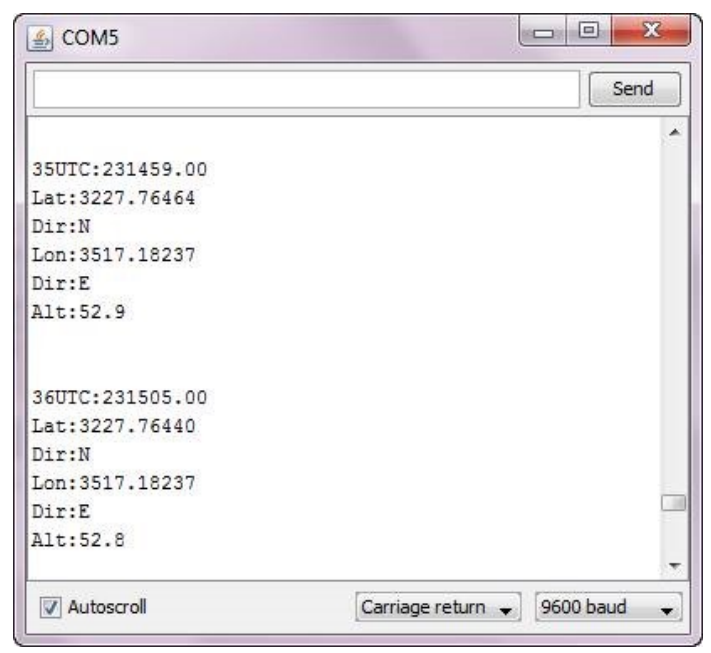

Fig. 13. The Position took by GPS Module

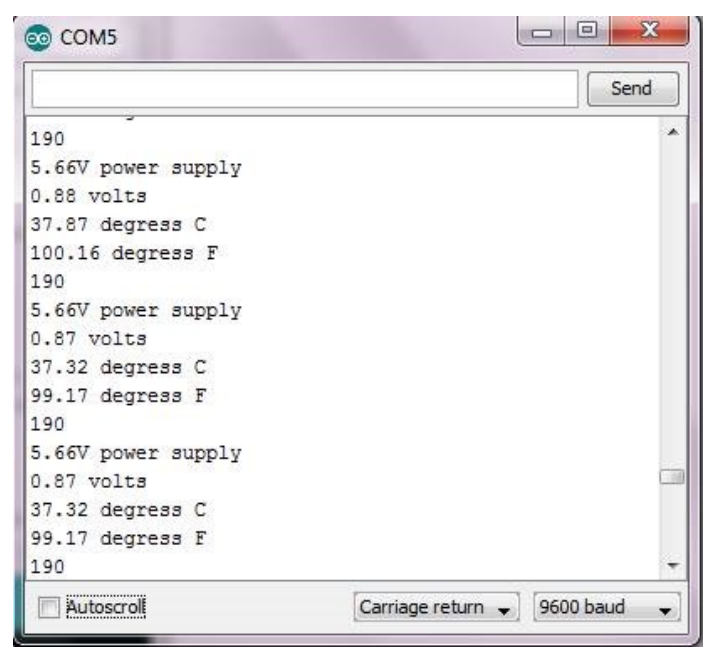

Fig. 14. LilyPad Temperature Sensor Readings

The sent SMS including Patients name, heart rate, body temperature, longitude and latitude of the position are exhibited on Figure Figure 15

And by using Google Map the location of the patient could be determined, and appeared in the SMS shown in Figure 16.

\section{CONCLUSION}

Nowadays, Globalization demands Smart cities, which involves many attributes and services, such as government services, Intelligent Transportation Systems (ITS), energy, healthcare, water and 


\section{John \\ Location: \\ 3227.75022N03517.21145}

E

\section{HR: 176}

temp33.4

\section{John \\ Location: \\ 3227.75433N03517.20867}

$\mathrm{E}$

HR: 67

temp22.1

Fig. 15. The SMS Exchanged by the System

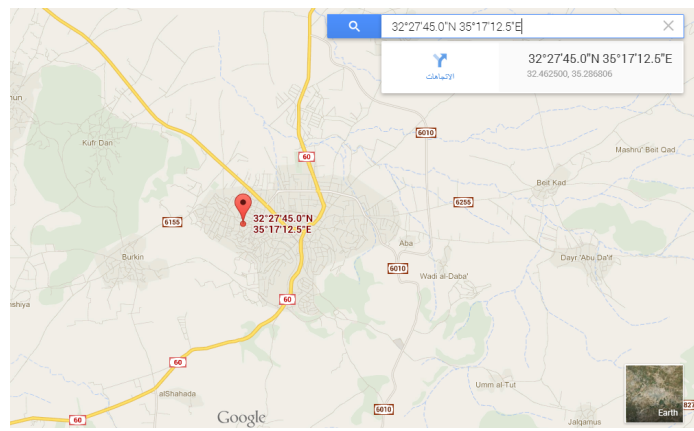

Fig. 16. Google Maps Patient Location

waste. while, healthcare is the most sensitive field to achieve the aim of smart city concept, by providing better life to society and build innovative and creative solutions, that are developed and enhanced by Smart system. the proposed model is designed to present sustainable medical interventions at manner time, by a smart system which is simple, using available world wide technologies, with a real time feedback.

The designed and experimented system, can significantly improve the quality of health services and reduce the total cost in healthcare by avoiding unnecessary hospitalizations and ensuring that those who need urgent care get it sooner.

The system model that is presented and discussed in this paper, can measure heartbeat rate and body temperature and communicate them in cases of extraordinary behaviors to supervision medical entities using GSM, GPS and web technologies to provocate immediate actions to rescue patient's life with potentiality in the future to add other vital factors measurements according to available sensor in the market which can achieve the objective of providing a reliable effective application for real time health monitoring and tracking.

The merit of this project relies on its future development targeted in two factors; first one: its multi-uses and services by making some modifications on the software, so many diseases and illnesses like Alzheimer, mental and motion patients could be benefited from this system; second factor: wireless technologies cloud be used to avoid wired connections which somehow may limit the patient mobility.

\section{REFERENCES}

[1] 4project. Pulse sensor. https://www.4project.co. il/product/pulse-sensor, 2015. [Online; accessed 29/11/2015]

[2] Angel. Lumo run - revolutionary smart running shorts. http://www.lumobodytech.com/, 2015. [Online; accessed 29/11/2015]

[3] K. Aziz, S. Tarapiah, S. H. Ismail, and S. Atalla. Smart real-time healthcare monitoring and tracking system using gsm/gps technologies. In 20163 rd MEC International Conference on Big Data and Smart City (ICBDSC), pages 1-7, March 2016.

[4] Kahtan Aziz, Saed Tarapiah, Mohanad Alsaedi, Salah Haj Ismail, and Shadi Atalla. Wireless sensor networks for road traffic monitoring. International Journal of Advanced Computer Science \& Applications, 1(6):265-270, 2015.

[5] Behance. Lifeline on behance. http://www. behance.net/ Gallery/LifeLinea/321119. 2015. [Online; accessed 29/11/2015].

[6] dash.co.il. Lilypad temperature sensor. http://goo.gl/ xnglsv, 2015. [Online; accessed 29/11/2015]

[7] Dfrobot. Gps/gprs/gsm module v2.0 (sku:tel0051) - robot wiki. http://goo.gl/r5Dm6u, 2015. [Online; accessed 29/11/2015].

[8] Wayne W Eckerson. Three tier client/server architectures: achieving scalability, performance, and efficiency in client/server applications. Open Information Systems, 3(20):46-50, 1995.

[9] WB Kannel and J Cobb. Left ventricular hypertrophy and mortality-results from the framingham study. Cardiology, 81(4-5):291-298, 1992.

[10] Patricia M Kearney, Megan Whelton, Kristi Reynolds, Paul Muntner, Paul K Whelton, and Jiang He. Global burden of hypertension: analysis of worldwide data. The lancet, 365(9455):217-223, 2005.

[11] Stephen S Lim, Theo Vos, Abraham D Flaxman, Goodarz Danaei, Kenji Shibuya, Heather Adair-Rohani, Mohammad A AlMazroa, Markus Amann, H Ross Anderson, Kathryn G Andrews, et al. A comparative risk assessment of burden of disease and injury attributable to 67 risk factors and risk factor clusters in 21 regions, 1990-2010: a systematic analysis for the global burden of disease study 2010. The lancet, 380(9859):2224-2260, 2013

[12] Alan D Lopez and CC Murray. The global burden of disease. Nat Med, 4(11):1241-1243, 1998.

[13] National High Blood Pressure Education Program et al. The seventh report of the joint national committee on prevention, detection, evaluation, and treatment of high blood pressure. National Heart, Lung, and Blood Institute (US), 2004. 
[14] Sarwant Singh. Smart cities - a \$1.5 trillion market opportunity. http://www.forbes. com/sites/sarwantsingh/2014/06/19/ smart-cities-a-1-5-trillion-market-opportunity/ 2015. [Online; accessed 29/11/2015].

[15] Mikhail St-Denis. Lifeline. http://www. mikhailstdenis.com/projects/personal_LifeLine. html 2015. [Online; accessed 29/11/2015].

[16] John S Steinhart and Stanley R Hart. Calibration curves for thermistors. In Deep Sea Research and Oceanographic Abstracts, volume 15, pages 497-503. Elsevier, 1968.

[17] Saed Tarapiah, Rajaa AbuHania, and Diana Jamal Islam Hindi. Applying web based gps/gprs ticketing and tracking mechanism to reduce traffic violation in developing countries. In The International Conference on Digital Information Processing, E-Business and Cloud Computing (DIPECC2013), pages 102-106. The Society of Digital Information and Wireless Communication, 2013.

[18] Saed Tarapiah and Shadi Atalla. Public transportation management system based on gps/wifi and open street maps. Editorial Preface, 6(1), 2015.

[19] Saed Tarapiah, Shadi Atalla, and Rajaa AbuHania. Smart onboard transportation management system using gps/gsm/gprs technologies to reduce traffic violation in developing countries. International Journal of Digital Information and Wireless Communications (IJDIWC), 3(4):430-439, 2013.

[20] Saed Tarapiah, Shadi Atalla, and B Alsayid. Smart on-board transportation management system geo-casting featured. In Computer Applications and Information Systems (WCCAIS), 2014 World Congress on, pages 1-6. IEEE, 2014.

[21] Saed Tarapiah, Shadi Atalla, Nooraldin Muala, and Sundos Tarabeh. Offline public transportation management system based on gps/wifi and open street maps. In Computational Intelligence, Communication Systems and Networks (CICSyN), 2014 Sixth International Conference on, pages 182185. IEEE, 2014.

[22] Saed Tarapiah, Kahtan Aziz, and Shadi Atalla. Common radio resource management algorithms in heterogeneous wireless networks with kpi analysis. International Journal of Advanced Computer Science \& Applications, 1(6):53-58, 2015.

[23] Saed Tarapiah, Kahtan Aziz, and Shadi Atalla. Radio resource management in heterogeneous networks, functional models and implementation requirements. International Journal of Computer Applications, 127(16):1-4, 2015.

[24] Thepu. how mobile is making us healthier. http://thepu.sh/trends/ take-two-wearables-and-call-me-in-the-morning-how-mobile-is-making-us-healthier/ 2015. [Online; accessed 29/11/2015].

[25] Arduino Uno. Arduino - arduinoboarduno. https://www. arduino.cc/en/Main/ArduinoBoardUno, 2015. [Online; accessed 29/11/2015]. 